\title{
The impact of the health condition of oaks from the Krotoszyn Plateau on the mechanical properties of timber structures
}

\author{
Jarosław Siwiński ${ }^{1}$, Katarzyna Kubiak ${ }^{2,3} \bowtie$, Miłosz Tkaczyk ${ }^{2}$, Anna Mazur ${ }^{3}$, Ryszard Rekucki $^{1}$ \\ ${ }^{1}$ Military University of Technology, Faculty of Civil Engineering and Geodesy, Gen. S. Kaliskiego 2, 00-908 Warsaw, \\ Poland \\ ${ }^{2}$ Forest Research Institute, Department of Forest Protection, Braci Leśnej 3, Sękocin Stary, 05-090 Raszyn, Poland, \\ phone: +4822 7153823, e-mail:k.kubiak@ibles.waw.pl \\ ${ }^{3}$ Institute of Aviation, Remote Sensing Department, al. Krakowska 110/114, 02-256 Warsaw, Poland
}

\section{Abstract}

The study was conducted to perform a comparative analysis of the mechanical properties of wood samples derived from oaks in the Krotoszyn Plateau, which depend on the health state of the trees. Strength parameters of oak wood were calculated for selected diseased and healthy trees (according to the Roloff classification). The study was conducted by a modified method described in the standard Polish Norm PN EN 408+ A1: 2012. For testing, prior selection of wood samples showed that more wood samples of diseased trees compared with those of healthy oaks did not fulfil the Polish standard requirements. According to the method used, the average results of strength tests of timber structures from healthy oaks exhibited higher strength parameters than those of the diseased trees.

\section{KeY WORDS}

health condition, Krotoszyn Plateau, mechanical properties of timber structures, oak

\section{INTRODUCTION}

From the second half of the $20^{\text {th }}$ century to the present, the oak decline phenomenon was observed on a large scale over all of Europe (Delatour 1983), as well as in California and Oregon State in North America (Wargo 1993). Reports of this phenomenon were also noted in Poland, with the highest intensity in the so-called 'Krotoszyn Plateau' (West Poland, Wielkopolska District, State Forest in Poznań). The main causes of this phenomenon are still being discussed amongst the sci- entific community and practitioners. In most cases, it was explained by a direct effect of pathogenic organisms that affect weakened trees (Oszako 2007). Some researchers stated the hypotheses in which they included a disadvantageous impact of individual abiotic and biotic factors (Dreyer and Aussenac 1996; Czech et al. 1998). Many secondary pests, such as Agrilus spp. insects or Hypoxylon spp. fungi, may contribute to the direct dieback of trees (Vannini 1991). Another causal agent in recent years includes infections with pathogens from the Phytophthora genus, which in many areas of 
the world causes serious root and other damages of oaks (Jung et al. 1996, 1999, 2002; Vettraino et al. 2002; Oszako and Orlikowski 2005; Oszako et al. 2007). The aim of this work was to check whether visible deterioration of trees (observed by evaluation of the crowns) affected the strength parameters of timber structures. For that purpose, tests of wood structure strength were performed and compared with the samples collected from both trees with visible signs of degraded health conditions and with those from healthy trees.

\section{Material AND MEthods}

Strength tests were carried out on a ZDN-10 endurance machine in the laboratory of the Military University of Technology. Tests were conducted according to the Polish Norm PN-EN 408 + A1 2012 'Timber structures: Structural timber and glued laminated timber - Determination of some physical and mechanical properties', which was modified to carry out comparative

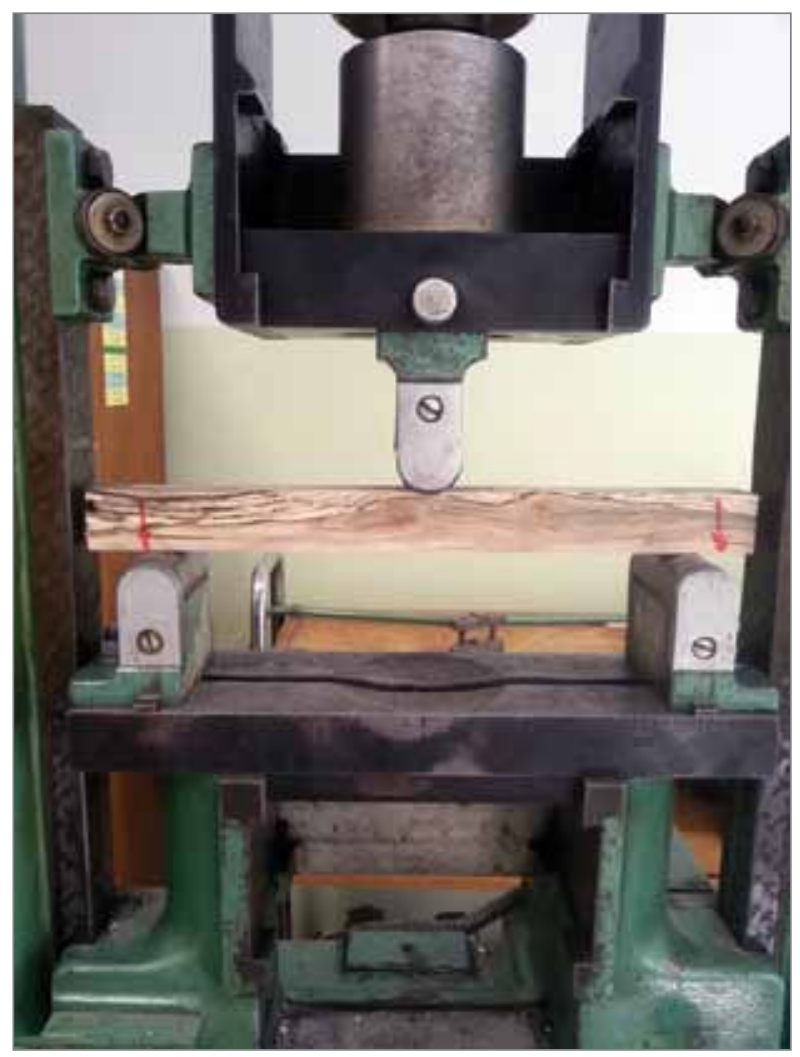

Figure 1. Static outline of sample support and load analyses. The analyses consisted of the attempt to bend prepared laboratory samples until obtaining a decline in the strength indicated on the machine manometer, that is, material destruction of individual test samples. Sample load was presented in Figure 1. The study was conducted in the laboratory room with air temperature of $24.4^{\circ} \mathrm{C}$ and relative air humidity of $52.0 \%$. Before carrying out the test, geometrical parameters and the humidity of timber structures samples were controlled using the resistance method. Before measurement and the cutting of short laboratory samples from primary samples, the samples were exposed to air conditioning at a temperature of $20^{\circ} \mathrm{C}$ for a period of 30 days. The samples were determined and selected so that their fibres ran parallel to the axes of beams, with growth rings parallel to one of the side plains. The results of strength of the timber structure were set based on the relationship (Polish Norm PN-EN 408+ A1 2012)

$$
R=\frac{3 P L}{2 b h^{2}}
$$

where:

$P$ - maximum load read from the instrument, expressed in $\mathrm{N}$,

$L$ - support width of the test sample in $\mathrm{mm}$,

$b$ - average width of the sample, being an average of three width measurement, in $\mathrm{mm}$,

$h$-average height of the sample, being an average of three height measurements, in $\mathrm{mm}$.

The results of strength of the timber structure $(R)$ were calculated, and they accounted for the real humidity of individual samples according to formula (2). The obtained strength of the timber structure $R_{d}$ accounts for the difference in humidity in relation to a norm value of 12\% (Polish Norm PN-EN 408: A1 2012; Szymański 2003).

$$
R_{d}=R[1+\alpha(w-12)]
$$

where:

$\alpha$ - rate with value of 0.04 ,

$w$ - measured humidity of the sample, expressed in $\%$.

Studies were carried out in accordance with norm procedures. Results were limited to the number of test samples indicated in Tables 1 and 2. 


\begin{tabular}{|c|c|c|c|c|c|c|c|c|c|c|c|c|c|c|c|c|c|c|c|}
\hline \multicolumn{2}{|c|}{ 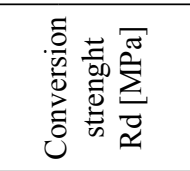 } & $\underset{0}{8}$ & $\stackrel{\substack{i \\
N}}{N}$ & in & $\begin{array}{l}\hat{b} \\
\text { á }\end{array}$ & $\hat{\alpha}$ & $\frac{n}{a}$ & $\frac{n}{\dot{a}}$ & $\begin{array}{l}\infty \\
\infty \\
\infty \\
\infty\end{array}$ & $\begin{array}{l}\stackrel{0}{n} \\
\stackrel{T}{T}\end{array}$ & $\frac{2}{a}$ & $\begin{array}{l}= \\
\infty\end{array}$ & ì & $\hat{\sigma}$ & $\begin{array}{l}\vec{\infty} \\
\hat{n} \\
n\end{array}$ & $\begin{array}{l}\stackrel{\infty}{+} \\
\dot{\infty}\end{array}$ & $\begin{array}{l}\infty \\
\infty \\
\dot{\infty}\end{array}$ & $\begin{array}{l}n \\
n \\
\infty \\
\infty\end{array}$ & 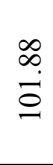 \\
\hline \multicolumn{2}{|c|}{ 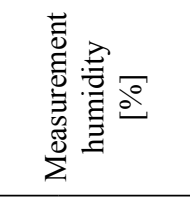 } & $\stackrel{\infty}{0}$ & $\begin{array}{l}\hat{b} \\
\text { I }\end{array}$ & 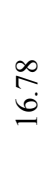 & $\begin{array}{l}\text { त्र } \\
\text { ॄे }\end{array}$ & 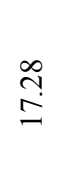 & $\stackrel{n}{n}$ & ก̋? & 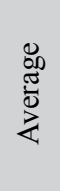 & $\begin{array}{l}\stackrel{\partial}{\circ} \\
\dot{ \pm}\end{array}$ & $\begin{array}{l}= \\
\ddot{m}\end{array}$ & $\underset{\infty}{\stackrel{2}{\infty}}$ & $\begin{array}{l}6 \\
0 \\
\infty\end{array}$ & $\stackrel{尺}{=}$ & $\begin{array}{l}\stackrel{\partial}{0} \\
\infty \\
\infty\end{array}$ & $\stackrel{2}{?}$ & 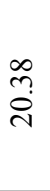 & $\stackrel{\nabla}{\Xi}$ & $\begin{array}{l}0 \\
\infty \\
\sigma\end{array}$ \\
\hline \multicolumn{2}{|c|}{ 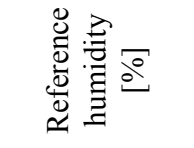 } & $\begin{array}{l}8 \\
\text { i }\end{array}$ & $\begin{array}{l}8 \\
\text { i }\end{array}$ & $\begin{array}{l}8 \\
\text { i }\end{array}$ & $\begin{array}{l}8 \\
\text { i }\end{array}$ & $\begin{array}{l}8 \\
i\end{array}$ & $\begin{array}{l}8 \\
i\end{array}$ & $\begin{array}{l}8 \\
\text { i }\end{array}$ & & $\begin{array}{l}\stackrel{8}{0} \\
\stackrel{i}{-}\end{array}$ & $\begin{array}{l}8 \\
\text { i }\end{array}$ & $\begin{array}{l}8 \\
\text { I }\end{array}$ & $\begin{array}{l}8 \\
i\end{array}$ & $\begin{array}{l}8 \\
1 \\
1\end{array}$ & $\begin{array}{l}8 \\
i \\
i\end{array}$ & $\begin{array}{l}8 \\
1 \\
1\end{array}$ & $\begin{array}{l}8 \\
\text { i }\end{array}$ & $\begin{array}{l}8 \\
\text { I }\end{array}$ & $\begin{array}{l}8 \\
i \\
\text { i }\end{array}$ \\
\hline \multicolumn{2}{|c|}{ 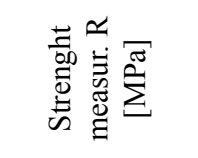 } & $\stackrel{n}{n}$ & $\stackrel{\text { in }}{n}$ & $\begin{array}{l}\infty \\
0 \\
0\end{array}$ & $\begin{array}{l}8 \\
8 \\
8\end{array}$ & กิ? & $\begin{array}{l}\text { D } \\
i n\end{array}$ & $\stackrel{+}{\stackrel{0}{\circ}}$ & & $\begin{array}{l}\vec{\infty} \\
\infty \\
\infty \\
0\end{array}$ & $\underset{n}{\tilde{\sigma}}$ & $\begin{array}{l}3 \\
\vdots \\
a \\
a\end{array}$ & 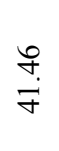 & $\stackrel{n}{\frac{n}{\infty}}$ & $\stackrel{\grave{n}}{\tilde{\gamma}}$ & $\stackrel{n}{m}$ & $\begin{array}{l}\mathscr{n} \\
\text { గె }\end{array}$ & $\begin{array}{l}8 \\
\infty \\
\infty \\
\infty\end{array}$ & $\stackrel{\vec{\exists}}{\Xi}$ \\
\hline \multicolumn{2}{|c|}{ 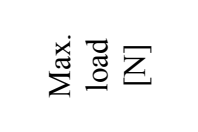 } & $\begin{array}{l}8 \\
8 \\
0 \\
0 \\
0 \\
n\end{array}$ & $\begin{array}{l}8 \\
8 \\
8 \\
\dot{0} \\
\qquad\end{array}$ & 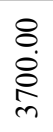 & $\begin{array}{l}8 \\
0 \\
0 \\
0 \\
0 \\
0\end{array}$ & 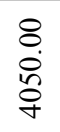 & $\begin{array}{l}8 \\
\stackrel{0}{0} \\
\stackrel{7}{7}\end{array}$ & $\begin{array}{l}8 \\
\stackrel{0}{0} \\
\stackrel{7}{7}\end{array}$ & & $\begin{array}{l}8 \\
8 \\
8 \\
0\end{array}$ & $\begin{array}{l}8 \\
\dot{8} \\
\infty \\
-1\end{array}$ & $\begin{array}{l}8 \\
\stackrel{8}{8} \\
\stackrel{8}{+} \\
\text { ণ }\end{array}$ & $\stackrel{8}{\circ}$ & $\begin{array}{l}8 \\
\vdots \\
\vdots \\
2\end{array}$ & $\begin{array}{l}8 \\
\stackrel{0}{0} \\
0\end{array}$ & $\begin{array}{l}8 \\
\dot{n} \\
\stackrel{n}{N}\end{array}$ & $\begin{array}{l}8 \\
\dot{8} \\
8 \\
\\
\end{array}$ & $\begin{array}{l}8 \\
\dot{8} \\
\stackrel{5}{+}\end{array}$ & 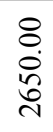 \\
\hline \multicolumn{2}{|c|}{ 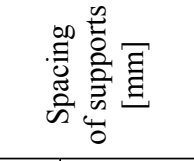 } & 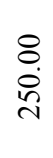 & 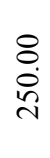 & $\begin{array}{l}8 \\
\stackrel{0}{0} \\
\stackrel{2}{1}\end{array}$ & $\begin{array}{l}8 \\
0 \\
\stackrel{0}{2}\end{array}$ & $\begin{array}{l}8 \\
\dot{0} \\
i\end{array}$ & $\begin{array}{l}8 \\
0 \\
0 \\
i\end{array}$ & $\begin{array}{l}8 \\
\stackrel{0}{0} \\
\stackrel{1}{1}\end{array}$ & & $\begin{array}{l}8 \\
\stackrel{i}{1}\end{array}$ & $\begin{array}{l}8 \\
\dot{8} \\
\dot{8}\end{array}$ & $\begin{array}{l}8 \\
\dot{8} \\
\dot{8}\end{array}$ & $\begin{array}{l}8 \\
\stackrel{+}{+}\end{array}$ & $\begin{array}{l}8 \\
0 \\
\dot{0}\end{array}$ & $\begin{array}{l}8 \\
\stackrel{+}{+}\end{array}$ & $\begin{array}{l}8 \\
\dot{8} \\
\stackrel{i}{1}\end{array}$ & $\begin{array}{l}8 \\
\stackrel{1}{+}\end{array}$ & $\begin{array}{l}8 \\
\dot{8} \\
\dot{8}\end{array}$ & $\begin{array}{l}8 \\
\dot{8} \\
\stackrel{i}{1}\end{array}$ \\
\hline \multirow{4}{*}{$\begin{array}{l}0 \\
1 \\
1 \\
\frac{1}{0} \\
0 \\
3\end{array}$} & 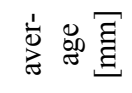 & $\begin{array}{l}0 \\
i n \\
\infty \\
\infty\end{array}$ & $\hat{a}$ & $\begin{array}{l}\infty \\
n \\
\infty \\
i\end{array}$ & $\begin{array}{l}0 \\
\stackrel{\sim}{0} \\
\infty \\
\sim\end{array}$ & $\begin{array}{l}\hat{\sim} \\
\stackrel{\sim}{N}\end{array}$ & 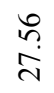 & 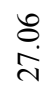 & & $\vec{m}$ & $\stackrel{\circ}{2}$ & $\frac{5}{9}$ & 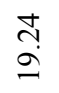 & $\begin{array}{l}0 \\
0 \\
0\end{array}$ & $\stackrel{\sim}{2}$ & 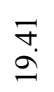 & $\stackrel{n}{\stackrel{2}{\leftrightarrows}}$ & $\hat{n}$ & $\begin{array}{l}\infty \\
\stackrel{\infty}{\infty} \\
\infty\end{array}$ \\
\hline & 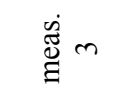 & 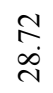 & $\begin{array}{l}\overrightarrow{0} \\
\infty \\
\dot{d}\end{array}$ & $\begin{array}{l}\overrightarrow{0} \\
\infty \\
\stackrel{i}{d}\end{array}$ & $\underset{\infty}{\infty}$ & $\underset{\hat{\sim}}{\hat{\sim}}$ & $\underset{\stackrel{\sim}{\sim}}{\stackrel{f}{N}}$ & $\begin{array}{l}2 \\
\dot{d}\end{array}$ & & $\begin{array}{l}\infty \\
\infty \\
\infty\end{array}$ & $\begin{array}{l}\circ \\
\infty \\
\infty \\
-1\end{array}$ & $\stackrel{2}{2}$ & $\stackrel{?}{\stackrel{2}{2}}$ & $\stackrel{9}{9}$ & $\stackrel{?}{\stackrel{9}{\sigma}}$ & $\stackrel{n}{2}$ & $\begin{array}{l}\tilde{b} \\
\infty \\
\infty\end{array}$ & $\begin{array}{l}\stackrel{9}{a} \\
\stackrel{2}{a}\end{array}$ & $\stackrel{\infty}{\infty}$ \\
\hline & $\dot{\mathscr{\Xi}} \sim$ & $\begin{array}{l}\hat{b} \\
\infty \\
\infty\end{array}$ & $\frac{8}{i}$ & $\begin{array}{l}\text { ọ } \\
\text { aे }\end{array}$ & $\begin{array}{l}\infty \\
n \\
\infty \\
\infty\end{array}$ & $\begin{array}{l}\infty \\
\stackrel{\sim}{\sim}\end{array}$ & $\begin{array}{l}8 \\
\infty \\
\infty \\
i\end{array}$ & $\underset{\stackrel{+}{\sim}}{\stackrel{\sim}{N}}$ & & $\begin{array}{l}\stackrel{+}{+} \\
\stackrel{2}{2}\end{array}$ & $\begin{array}{l}\stackrel{2}{2} \\
\stackrel{2}{2}\end{array}$ & 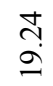 & $\ddot{\circ}$ & $\begin{array}{l}\infty \\
\infty \\
\infty\end{array}$ & $\stackrel{m}{m}$ & $\begin{array}{l}8 \\
\stackrel{0}{9}\end{array}$ & 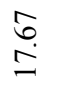 & 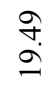 & $\begin{array}{l}0 \\
\stackrel{\infty}{\infty}\end{array}$ \\
\hline & $\begin{array}{l}\dot{\Xi} \\
\stackrel{\Xi}{\Xi}-\end{array}$ & 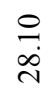 & $\begin{array}{l}8 \\
\stackrel{\infty}{\infty} \\
\infty\end{array}$ & $\begin{array}{l}\stackrel{n}{q} \\
\infty \\
\sim \\
\sim\end{array}$ & $\begin{array}{l}\infty \\
\stackrel{\infty}{\infty} \\
\stackrel{\sim}{d}\end{array}$ & 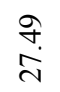 & 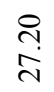 & $\underset{\stackrel{\Delta}{\sim}}{\stackrel{\Delta}{\sim}}$ & & $\begin{array}{l}\infty \\
\stackrel{\infty}{2}\end{array}$ & $\hat{\alpha}$ & $\begin{array}{l}0 \\
9 \\
9\end{array}$ & $\begin{array}{c}\tilde{\sigma} \\
\stackrel{-}{a}\end{array}$ & $\overrightarrow{\widetilde{T}}$ & $\begin{array}{l}\vec{\infty} \\
\stackrel{a}{a}\end{array}$ & $=$ & $\underline{\hat{a}}$ & $\stackrel{\tilde{丶}}{\stackrel{2}{2}}$ & $\stackrel{\sim}{\stackrel{2}{2}}$ \\
\hline \multirow{4}{*}{ 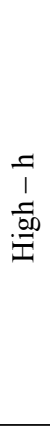 } & 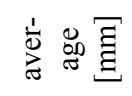 & $\stackrel{\sqrt[n]{n}}{\sim}$ & ते & $\begin{array}{l}\stackrel{i}{\infty} \\
\stackrel{N}{N}\end{array}$ & 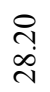 & $\underset{\infty}{\infty}$ & $\begin{array}{l}\stackrel{a}{0} \\
\infty \\
\sim\end{array}$ & $\begin{array}{l}\hat{o} \\
\infty \\
\infty\end{array}$ & & $\stackrel{\square}{\circ}$ & 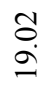 & $\begin{array}{l}\vec{\sigma} \\
\stackrel{0}{0}\end{array}$ & 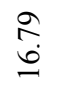 & $\vec{\sim}$ & $\vec{m}$ & $\stackrel{\infty}{a}$ & సે̀ & $\begin{array}{l}\stackrel{+}{n} \\
\stackrel{2}{2}\end{array}$ & $\begin{array}{l}\stackrel{P}{2} \\
\stackrel{2}{2}\end{array}$ \\
\hline & 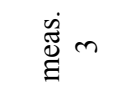 & $\frac{d}{\stackrel{n}{\Lambda}}$ & $\begin{array}{l}\stackrel{2}{\pi} \\
\stackrel{1}{1}\end{array}$ & $\begin{array}{l}\sim \\
\substack{\infty \\
\infty}\end{array}$ & 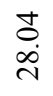 & $\stackrel{m}{\infty}$ & $\underset{\substack{n \\
\infty}}{\infty}$ & $\stackrel{n}{\infty}$ & & $\stackrel{8}{\infty}$ & $\begin{array}{l}\stackrel{\partial}{\infty} \\
\infty\end{array}$ & $\begin{array}{l}8 \\
0 \\
0\end{array}$ & $\stackrel{\infty}{n}$ & $\frac{n}{\infty}$ & $\begin{array}{l}\infty \\
\infty \\
\infty \\
-\infty\end{array}$ & $\vec{m}$ & $\begin{array}{l}\infty \\
\infty \\
\infty\end{array}$ & $\begin{array}{l}\infty \\
\stackrel{n}{2} \\
\stackrel{2}{2}\end{array}$ & $\frac{9}{2}$ \\
\hline & 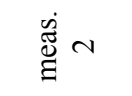 & $\begin{array}{l}\infty \\
\dot{0} \\
\stackrel{i}{N}\end{array}$ & $\begin{array}{l}\hat{\sigma} \\
\infty \\
i\end{array}$ & 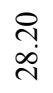 & 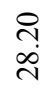 & $\begin{array}{l}0 \\
\underset{\infty}{\sim} \\
\stackrel{\sim}{N}\end{array}$ & $\begin{array}{l}\underset{\sim}{\tilde{N}} \\
\infty \\
\sim\end{array}$ & $\begin{array}{l}0 \\
\stackrel{0}{0} \\
\infty \\
i\end{array}$ & & $\begin{array}{l}\ddot{2} \\
\stackrel{0}{a}\end{array}$ & $\begin{array}{l}+ \\
\infty \\
\infty \\
\infty\end{array}$ & $\begin{array}{l}3 \\
0 \\
2\end{array}$ & $\stackrel{n}{n}$ & $\begin{array}{l}\stackrel{\infty}{\infty} \\
\infty \\
\infty\end{array}$ & 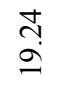 & $\stackrel{ \pm}{a}$ & $\stackrel{2}{\stackrel{9}{2}}$ & $\stackrel{\infty}{\stackrel{\infty}{\sigma}}$ & $\begin{array}{l}+ \\
\stackrel{+}{\circ}\end{array}$ \\
\hline & $\stackrel{\dot{\Xi}}{\stackrel{\Xi}{\Xi}}-$ & $\begin{array}{l}\tilde{d} \\
\stackrel{\sim}{\sim}\end{array}$ & $\begin{array}{l}\stackrel{+}{\sim} \\
\stackrel{i}{\sim}\end{array}$ & 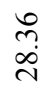 & $\begin{array}{l}0 \\
\substack{n \\
\infty \\
i}\end{array}$ & $\underset{\infty}{\infty}$ & $\stackrel{\vec{n}}{\stackrel{n}{n}}$ & $\stackrel{\overbrace{}}{\stackrel{\sim}{\sim}}$ & & $\hat{\circ}$ & તุ. & $\begin{array}{l}\infty \\
\stackrel{n}{2} \\
\stackrel{2}{2}\end{array}$ & $\begin{array}{l}\hat{\sigma} \\
\infty \\
\infty\end{array}$ & શે & $\begin{array}{l}\grave{a} \\
\stackrel{0}{a}\end{array}$ & $\begin{array}{l}\stackrel{\infty}{\circ} \\
\stackrel{9}{9}\end{array}$ & $\begin{array}{l}\text { ते } \\
\text { 2. }\end{array}$ & $\stackrel{\hat{N}}{\grave{2}}$ & $\begin{array}{l}\infty \\
\stackrel{0}{0} \\
\stackrel{\sim}{N}\end{array}$ \\
\hline \multirow{2}{*}{\multicolumn{2}{|c|}{$\dot{z}$}} & - & N & n & $\nabla$ & in & 6 & $r$ & & - & N & $m$ & $\nabla$ & $n$ & 0 & $r$ & $\infty$ & $a$ & 은 \\
\hline & & \multicolumn{7}{|c|}{ 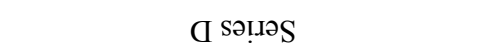 } & & \multicolumn{10}{|c|}{ W sət.઼ S } \\
\hline
\end{tabular}




\begin{tabular}{|c|c|c|c|c|c|c|c|c|c|c|c|c|c|c|c|c|c|c|c|c|c|c|}
\hline \multicolumn{2}{|c|}{ 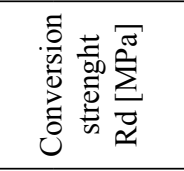 } & f̊ & $\stackrel{n}{n}$ & f̊ & $\begin{array}{l}\stackrel{\infty}{i} \\
i \\
i\end{array}$ & $\begin{array}{l}7 \\
8 \\
8\end{array}$ & $\begin{array}{l}\vec{b} \\
\dot{2}\end{array}$ & $\begin{array}{l}\hat{\sigma} \\
\grave{\alpha}\end{array}$ & $\begin{array}{l}\bar{Z} \\
\dot{\Sigma} \\
\infty\end{array}$ & $\stackrel{+}{\stackrel{\Delta}{\delta}}$ & $\begin{array}{l}n \\
o \\
i n\end{array}$ & 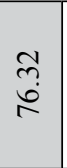 & $\begin{array}{l}\vec{b} \\
\infty \\
\infty\end{array}$ & $\begin{array}{r}\tilde{3} \\
\tilde{8}\end{array}$ & $\begin{array}{l}\tilde{\sigma} \\
\dot{\sigma}\end{array}$ & 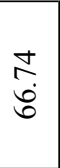 & $\begin{array}{l}0 \\
0 \\
\dot{q} \\
\dot{+}\end{array}$ & $\begin{array}{l}\infty \\
\infty \\
\infty \\
\infty\end{array}$ & $\begin{array}{l}0 \\
0 \\
0 \\
0 \\
0\end{array}$ & 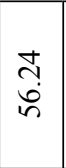 & 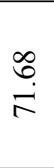 & $\frac{1}{8}$ \\
\hline \multicolumn{2}{|c|}{ 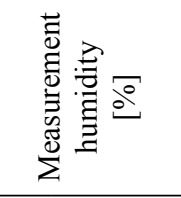 } & $\stackrel{\infty}{=}$ & oे & 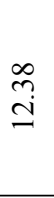 & 守 & $\begin{array}{l}\infty \\
0 \\
i \\
\end{array}$ & 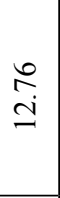 & $\stackrel{2}{\stackrel{0}{0}}$ & $\overrightarrow{\tilde{O}}$ & $\stackrel{\overbrace{}}{\stackrel{?}{=}}$ & $\stackrel{n}{\varrho}$ & 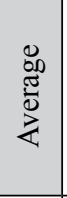 & $\begin{array}{l}0 \\
0 \\
\infty\end{array}$ & $\tilde{n}$ & 号 & $\stackrel{0}{\circ}$ & $\begin{array}{l}n \\
a \\
a\end{array}$ & $\stackrel{\infty}{\stackrel{\infty}{r}}$ & $\underset{\infty}{+}$ & $\begin{array}{l}n \\
\infty \\
a \\
a\end{array} \mid$ & 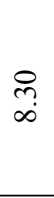 & $\stackrel{9}{=}$ \\
\hline \multicolumn{2}{|c|}{ 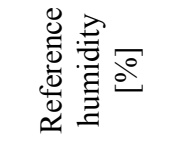 } & $\begin{array}{l}\stackrel{8}{0} \\
\text { i }\end{array}$ & $\begin{array}{l}8 \\
i \\
i\end{array}$ & $\begin{array}{l}8 \\
\text { I }\end{array}$ & $\begin{array}{l}8 \\
\text { i }\end{array}$ & $\begin{array}{l}8 \\
\text { I }\end{array}$ & $\begin{array}{l}8 \\
\text { i }\end{array}$ & $\begin{array}{l}8 \\
\dot{I} \\
\text { I }\end{array}$ & $\begin{array}{l}8 \\
\stackrel{0}{1} \\
\text { I }\end{array}$ & $\begin{array}{l}\stackrel{8}{ } \\
\text { i }\end{array}$ & $\begin{array}{l}\stackrel{8}{0} \\
\text { i }\end{array}$ & & $\begin{array}{l}8 \\
i \\
i\end{array}$ & $\begin{array}{l}\underset{8}{8} \\
\text { i }\end{array}$ & $\begin{array}{l}8 \\
\text { i }\end{array}$ & $\begin{array}{l}8 \\
\text { i }\end{array}$ & $\begin{array}{l}8 \\
i \\
\text { I }\end{array}$ & $\begin{array}{l}8 \\
i \\
i\end{array}$ & $\begin{array}{l}\stackrel{8}{ } \\
\text { i }\end{array}$ & $\begin{array}{l}8 \\
i \\
\text { I }\end{array}$ & $\begin{array}{l}\stackrel{8}{ } \\
\text { I }\end{array}$ & $\begin{array}{l}8 \\
\text { i }\end{array}$ \\
\hline \multicolumn{2}{|c|}{ 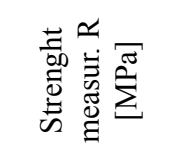 } & $\begin{array}{l}\infty \\
\dot{a} \\
\dot{a}\end{array}$ & $\begin{array}{l}\tilde{f} \\
\dot{b} \\
\infty\end{array}$ & $\stackrel{m}{n}$ & $\begin{array}{l}\bar{m} \\
\dot{\infty}\end{array}$ & 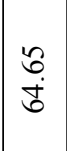 & 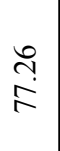 & $\begin{array}{l}\infty \\
\dot{\sigma} \\
\end{array}$ & \begin{tabular}{|l|}
$\infty$ \\
$n$ \\
0 \\
$\infty$
\end{tabular} \mid & $\stackrel{n}{i}$ & $\underset{n}{n}$ & & $\begin{array}{l}n \\
\dot{q}\end{array}$ & $\begin{array}{l}0 \\
\infty \\
0 \\
0\end{array}$ & $\begin{array}{l}\widetilde{1} \\
\dot{+} \\
\stackrel{0}{0}\end{array}$ & $\begin{array}{l}\dot{B} \\
\dot{i} \\
i\end{array}$ & $\begin{array}{l}m \\
\vec{q} \\
\dot{q}\end{array}$ & $\begin{array}{l}\stackrel{n}{f} \\
\tilde{\sigma} \\
\tilde{\theta}\end{array}$ & $\begin{array}{l}\infty \\
\infty \\
2 \\
\end{array}$ & $\frac{n}{n}$ & $\begin{array}{l}\dot{I} \\
\dot{\infty}\end{array}$ & $\begin{array}{l}0 \\
\infty \\
\infty \\
\infty\end{array}$ \\
\hline \multicolumn{2}{|c|}{ 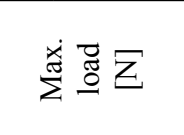 } & $\begin{array}{l}8 \\
8 \\
8 \\
8\end{array}$ & $\begin{array}{l}8 \\
8 \\
0 \\
0 \\
n\end{array}$ & \begin{tabular}{l}
8 \\
8 \\
8 \\
8 \\
\multirow{7}{7}{}
\end{tabular} & $\begin{array}{l}8 \\
0 \\
i n \\
\text { in }\end{array}$ & $\begin{array}{l}8 \\
8 \\
\dot{0} \\
\stackrel{+}{+} \\
\dot{7}\end{array}$ & 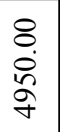 & 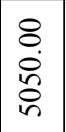 & \begin{tabular}{l|} 
\\
\\
$\dot{8}$ \\
$\dot{0}$ \\
$n$
\end{tabular} & $\begin{array}{l}8 \\
8 \\
0 \\
n \\
y\end{array}$ & $\begin{array}{l}8 \\
\dot{8} \\
\text { ळे }\end{array}$ & & 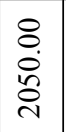 & $\begin{array}{l}8 \\
8 \\
8 \\
0\end{array}$ & $\begin{array}{l}8 \\
0 \\
0 \\
0 \\
i \\
\end{array}$ & $\begin{array}{l}8 \\
8 \\
\dot{8} \\
-\end{array}$ & \begin{tabular}{|l|}
8 \\
$\dot{8}$ \\
$\stackrel{1}{-}$ \\
\end{tabular} & \begin{tabular}{l|}
8 \\
$\dot{0}$ \\
$\stackrel{5}{7}$ \\
\end{tabular} & $\begin{array}{l}8 \\
8 \\
8 \\
\end{array}$ & \begin{tabular}{|l|} 
\\
\\
$\dot{8}$ \\
- \\
-
\end{tabular} & $\begin{array}{l}8 \\
\circ \\
\infty \\
\infty\end{array}$ & 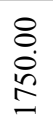 \\
\hline \multicolumn{2}{|c|}{ 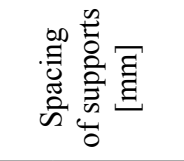 } & $\begin{array}{l}8 \\
\ddot{\circ} \\
i\end{array}$ & $\begin{array}{l}8 \\
0 \\
0 \\
i\end{array}$ & $\begin{array}{l}8 \\
\ddot{1} \\
i\end{array}$ & $\begin{array}{l}8 \\
0 \\
\check{n} \\
\end{array}$ & $\begin{array}{l}8 \\
\dot{0} \\
i n \\
\end{array}$ & $\begin{array}{l}8 \\
0 \\
i \\
i\end{array}$ & $\begin{array}{l}8 \\
0 \\
\dot{2} \\
i\end{array}$ & $\begin{array}{l}8 \\
8 \\
\dot{0} \\
\end{array}$ & 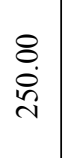 & $\begin{array}{l}8 \\
\ddot{\dot{a}} \\
i\end{array}$ & & $\begin{array}{l}8 \\
\dot{8} \\
\dot{2}\end{array}$ & $\begin{array}{l}8 \\
\ddot{8} \\
\dot{8}\end{array}$ & $\begin{array}{l}8 \\
\dot{0} \\
\dot{0}\end{array}$ & $\begin{array}{l}\stackrel{8}{8} \\
\ddot{\leftrightarrow} \\
\stackrel{\sim}{1}\end{array}$ & $\begin{array}{l}8 \\
8 \\
\dot{8} \\
\stackrel{1}{1}\end{array}$ & $\begin{array}{l}8 \\
\dot{0} \\
\dot{8} \\
\end{array}$ & $\begin{array}{l}8 \\
\dot{0} \\
\dot{0}\end{array}$ & $\begin{array}{l}8 \\
\dot{0} \\
\dot{8}\end{array}$ & $\begin{array}{l}8 \\
\ddot{8} \\
\stackrel{0}{1}\end{array}$ & $\begin{array}{l}8 \\
\stackrel{8}{8}\end{array}$ \\
\hline \multirow{4}{*}{$\begin{array}{l}0 \\
1 \\
1 \\
\frac{1}{0} \\
0 \\
3\end{array}$} & 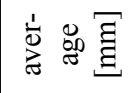 & $\underset{\stackrel{?}{\sim}}{\stackrel{\sim}{\sim}}$ & $\begin{array}{l}\vec{n} \\
\infty \\
\infty\end{array}$ & $\begin{array}{l}n \\
\infty \\
\infty \\
i\end{array}$ & $\begin{array}{l}n \\
i \\
\infty \\
i\end{array}$ & $\begin{array}{l}n \\
i \\
\infty \\
\infty\end{array}$ & $\begin{array}{l}n \\
n \\
\infty \\
i \\
i\end{array}$ & $\begin{array}{l}8 \\
0 \\
\dot{1}\end{array}$ & $\begin{array}{l}\tilde{n} \\
\infty \\
\sim\end{array}$ & ले & ?̊. & & $\begin{array}{l}\stackrel{\Upsilon}{2} \\
\stackrel{2}{2}\end{array}$ & $\stackrel{-}{a}$ & $\begin{array}{l}\vec{\infty} \\
\stackrel{-}{-}\end{array}$ & $\stackrel{\stackrel{m}{2}}{\stackrel{2}{=}}$ & $\mid \begin{array}{l}\infty \\
0 \\
i \\
i \\
\tilde{n}\end{array}$ & $\stackrel{7}{9}$ & $\stackrel{n}{\varrho}$ & $\begin{array}{l}8 \\
\dot{0} \\
-\end{array}$ & $\begin{array}{l}\stackrel{\Re}{\stackrel{\infty}{\infty}} \\
\stackrel{\infty}{-}\end{array}$ & $\begin{array}{l}\vec{J} \\
\infty \\
-\end{array}$ \\
\hline & $\dot{\vec{d}} n$ & ڤ్ & $\begin{array}{l}\alpha \\
\alpha \\
\infty \\
\sim \\
\sim\end{array}$ & 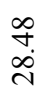 & $\underset{\substack{\infty \\
\stackrel{\infty}{\sim}}}{\stackrel{p}{0}}$ & $\begin{array}{l}n \\
n \\
\infty \\
n\end{array}$ & $\begin{array}{l}+ \\
\infty \\
\infty \\
\infty \\
\sim\end{array}$ & $\underset{\Delta}{ \pm}$ & $\begin{array}{l}\infty \\
i \\
\infty \\
\sim\end{array}$ & $=$ & ֻั. & & $\frac{n}{a}$ & $\stackrel{1}{9}$ & $\begin{array}{l}\dot{t} \\
\stackrel{0}{-}\end{array}$ & $\begin{array}{l}\stackrel{\infty}{\sim} \\
\stackrel{2}{\sim}\end{array}$ & $\begin{array}{l}0 \\
\stackrel{1}{i} \\
\stackrel{i}{1}\end{array}$ & $\begin{array}{l}\infty \\
\stackrel{\infty}{\infty} \\
-\end{array}$ & $\begin{array}{l}0 \\
\stackrel{n}{2} \\
a\end{array}$ & $\stackrel{2}{a}$ & $\hat{n}$ & $\frac{a}{\infty}$ \\
\hline & 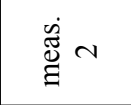 & $\begin{array}{l}\text { oे } \\
\text { iे }\end{array}$ & $\begin{array}{l}0 \\
n \\
\infty \\
i\end{array}$ & $\stackrel{n}{\stackrel{n}{i}}$ & $\frac{n}{\grave{s}}$ & $\begin{array}{l}\stackrel{\infty}{+} \\
\stackrel{\infty}{\sim} \\
\stackrel{\sim}{\sim}\end{array}$ & $\begin{array}{l}\hat{n} \\
\infty \\
i\end{array}$ & \begin{tabular}{l|}
$\Delta$ \\
$\Delta$ \\
$\Delta$ \\
\end{tabular} & 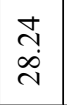 & $\begin{array}{l}\stackrel{\sim}{\leftarrow} \\
\stackrel{\sim}{\sim}\end{array}$ & $\begin{array}{l}\hat{n} \\
\text { ते }\end{array}$ & & 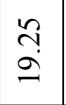 & $\stackrel{ \pm}{-}$ & $\stackrel{\hat{2}}{2}$ & 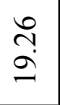 & 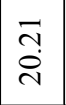 & సे & $\begin{array}{l}\stackrel{n}{g} \\
\stackrel{2}{a}\end{array}$ & \begin{tabular}{l|}
0 \\
$\infty$ \\
2 \\
2
\end{tabular} & 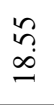 & $\underset{\stackrel{\mathcal{Y}}{\infty}}{\infty}$ \\
\hline & 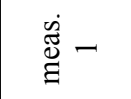 & $\begin{array}{l}n \\
\stackrel{n}{N}\end{array}$ & $\begin{array}{l}n \\
0 \\
\infty \\
i\end{array}$ & $\underset{\sim}{\infty}$ & 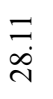 & $\begin{array}{c}\stackrel{p}{i} \\
i \\
i\end{array}$ & $\begin{array}{c}0 \\
\substack{n \\
\infty \\
i}\end{array}$ & $\stackrel{m}{\stackrel{n}{\sim}}$ & $\begin{array}{l}\infty \\
\infty \\
\infty \\
\sim\end{array}$ & $\begin{array}{l}n \\
\tilde{a} \\
\grave{2}\end{array}$ & $\vec{m}$ & & 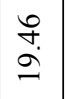 & $\begin{array}{l}\stackrel{0}{1} \\
\stackrel{2}{2}\end{array}$ & $\begin{array}{l}\ddot{i} \\
\ddot{i}\end{array}$ & \begin{tabular}{l}
\multirow{2}{*}{} \\
$\stackrel{2}{-}$
\end{tabular} & $\begin{array}{l}+ \\
\infty \\
\dot{\sim}\end{array}$ & $\begin{array}{l}\mathfrak{g} \\
\stackrel{2}{2}\end{array}$ & $\begin{array}{l}\tilde{c} \\
\stackrel{2}{2}\end{array}$ & $\stackrel{\Xi}{2}$ & $\underset{\hat{\infty}}{\infty}$ & $\begin{array}{l}\tilde{b} \\
\infty \\
\infty\end{array}$ \\
\hline \multirow{4}{*}{$\begin{array}{l}= \\
1 \\
\frac{1}{50} \\
3 \\
01\end{array}$} & 岕品志 & $\underset{\stackrel{n}{\sim}}{\stackrel{m}{\sim}}$ & $\begin{array}{l}a \\
\dot{0} \\
\infty \\
i\end{array}$ & $\begin{array}{l}\stackrel{\infty}{\infty} \\
\stackrel{\sim}{N}\end{array}$ & $\underset{\substack{+\stackrel{\infty}{\sim}}}{\stackrel{\infty}{\sim}}$ & $\begin{array}{l}\stackrel{0}{0} \\
\stackrel{0}{0}\end{array}$ & $\begin{array}{l}\text { ô. } \\
\grave{2}\end{array}$ & 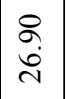 & $\begin{array}{l}\overrightarrow{2} \\
\grave{2}\end{array}$ & $\begin{array}{l}\stackrel{\rho}{+} \\
\infty \\
\stackrel{\sim}{d}\end{array}$ & ণ্ণ & & $\begin{array}{l}n \\
\infty \\
\infty \\
\infty\end{array}$ & $\stackrel{n}{\dddot{2}}$ & $\begin{array}{l}\dot{t} \\
\stackrel{0}{-}\end{array}$ & $\begin{array}{l}\infty \\
0 \\
\infty \\
\infty\end{array}$ & $\begin{array}{l}\infty \\
2 \\
2 \\
2\end{array}$ & $\begin{array}{l}a \\
a \\
2\end{array}$ & $\stackrel{\Xi}{\infty}$ & $\begin{array}{l}\infty \\
\vdots \\
\end{array}$ & $\begin{array}{l}\hat{N} \\
\infty\end{array}$ & $\begin{array}{l}n \\
n \\
\infty\end{array}$ \\
\hline & 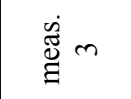 & $\frac{n}{\stackrel{n}{n}}$ & $\begin{array}{l}\dot{1} \\
\infty \\
\infty \\
\infty\end{array}$ & $\begin{array}{l}\infty \\
\infty \\
\stackrel{\sim}{\sim}\end{array}$ & 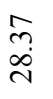 & $\stackrel{\vartheta}{\overrightarrow{0}}$ & $\begin{array}{l}\text { f. } \\
\text { ते }\end{array}$ & 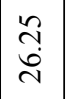 & $\begin{array}{l}\infty \\
\infty \\
\infty \\
\infty \\
\sim\end{array}$ & $\begin{array}{l}\infty \\
\infty \\
\stackrel{\sim}{\sim}\end{array}$ & in & & $\begin{array}{l}0 \\
0 \\
9 \\
-\end{array}$ & $\begin{array}{l}\stackrel{2}{n} \\
\stackrel{2}{=}\end{array}$ & $\underset{\stackrel{+}{*}}{\stackrel{2}{二}}$ & $\begin{array}{l}0 \\
i \\
\infty \\
\infty\end{array}$ & $\begin{array}{l}\check{2} \\
\stackrel{2}{2}\end{array}$ & $\begin{array}{l}\hat{6} \\
2 \\
2\end{array}$ & $\stackrel{?}{\stackrel{2}{I}}$ & 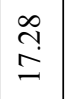 & 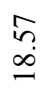 & $\stackrel{\infty}{\stackrel{\infty}{I}}$ \\
\hline & 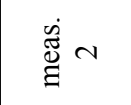 & $\underset{\stackrel{n}{\sim}}{\stackrel{?}{\sim}}$ & $\begin{array}{l}\infty \\
\infty \\
\infty \\
\infty \\
\sim\end{array}$ & $\underset{\substack{\infty \\
\stackrel{\infty}{\sim}}}{\stackrel{0}{1}}$ & $\begin{array}{l}n \\
n \\
\infty \\
i\end{array}$ & $\begin{array}{l}8 \\
\dot{0} \\
\dot{m}\end{array}$ & $\begin{array}{l}\alpha \\
\alpha \\
\alpha \\
\sim\end{array}$ & $\begin{array}{l}n \\
\hat{\sigma} \\
\hat{i}\end{array}$ & $\begin{array}{l}\tilde{a} \\
\grave{\lambda}\end{array}$ & 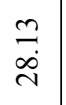 & $\begin{array}{l}\text { J̦ } \\
\stackrel{\text { ते }}{ }\end{array}$ & & $\begin{array}{l}\mathcal{T} \\
\stackrel{+}{+} \\
\infty \\
- \\
\end{array}$ & $\stackrel{+}{2}$ & $\stackrel{\infty}{\stackrel{\infty}{2}}$ & $\begin{array}{l}n \\
\tilde{n} \\
\infty\end{array}$ & \begin{tabular}{l|}
+ \\
$\infty$ \\
2 \\
2
\end{tabular} & $\begin{array}{l}\hat{a} \\
\infty \\
\infty\end{array}$ & $\begin{array}{l}\stackrel{2}{\infty} \\
\stackrel{-}{-}\end{array}$ & 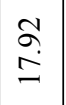 & $\begin{array}{l}8 \\
\infty \\
\infty\end{array}$ & $\begin{array}{l}\infty \\
\stackrel{\infty}{+} \\
\infty\end{array}$ \\
\hline & 宛 - & 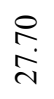 & $\begin{array}{l}8 \\
0 \\
\infty \\
i\end{array}$ & 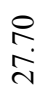 & $\begin{array}{l}\vec{n} \\
\infty \\
\infty\end{array}$ & $\begin{array}{l}\infty \\
\stackrel{2}{2} \\
\text { ते }\end{array}$ & $\begin{array}{c}\vec{i} \\
i \\
i\end{array}$ & 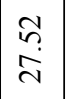 & $\frac{2}{\grave{2}}$ & 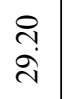 & ñ? & & $\begin{array}{l}0 \\
\underset{\infty}{0} \\
-1\end{array}$ & $\stackrel{\circ}{\stackrel{0}{2}}$ & $\stackrel{8}{\circ}$ & $\begin{array}{l}\check{a} \\
\stackrel{2}{-}\end{array}$ & $\begin{array}{l}0 \\
2 \\
2 \\
2\end{array}$ & $\exists$ & $\begin{array}{l}\bar{\sigma} \\
\infty \\
-\end{array}$ & $\begin{array}{l}n \\
\infty \\
\infty \\
\infty\end{array}$ & $\stackrel{m}{2}$ & $\begin{array}{l}0 \\
\text { ?ִ } \\
\stackrel{9}{=}\end{array}$ \\
\hline \multirow{2}{*}{\multicolumn{2}{|c|}{$\dot{z}$}} & - & 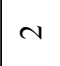 & $n$ & $\nabla$ & in & 0 & $r$ & $\infty$ & $a$ & $\circ$ & & - & N & $n$ & $\nabla$ & in & 0 & $r$ & $\infty$ & $a$ & 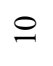 \\
\hline & & \multicolumn{10}{|c|}{ C sə̣..əs } & \multicolumn{11}{|c|}{ W sə઼..əs } \\
\hline
\end{tabular}




\begin{tabular}{|c|c|c|c|c|c|c|c|c|c|c|c|c|c|c|c|}
\hline \multicolumn{2}{|c|}{ 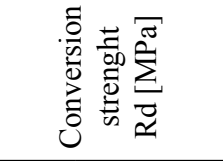 } & oे & $\begin{array}{l}3 \\
\dot{a}\end{array}$ & ळे & $\frac{n}{n}$ & $\frac{n}{8}$ & $\underset{\infty}{\infty}$ & $\begin{array}{l}\stackrel{R}{T} \\
\stackrel{i}{n}\end{array}$ & $\frac{2}{2}$ & $=$ & 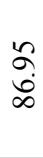 & $\begin{array}{l}\stackrel{\infty}{\oplus} \\
\dot{\infty}\end{array}$ & $\begin{array}{l}\infty \\
\infty \\
\infty\end{array}$ & $\begin{array}{l}\infty \\
\stackrel{\infty}{0} \\
\stackrel{0}{0}\end{array}$ & $\begin{array}{c} \pm \\
m \\
\infty \\
\infty\end{array}$ \\
\hline \multicolumn{2}{|c|}{ 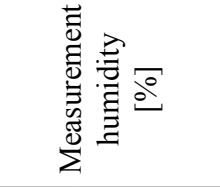 } & مै & సֶ. & $\stackrel{\infty}{\stackrel{N}{I}}$ & $\stackrel{?}{\stackrel{n}{=}}$ & กิ? & 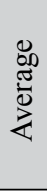 & $\begin{array}{l}\stackrel{\partial}{\circ} \\
\dot{ \pm}\end{array}$ & $=$ & $\stackrel{2}{\infty}$ & $\stackrel{尺}{\Xi}$ & $\stackrel{?}{?}$ & $\stackrel{\infty}{\stackrel{\infty}{*}}$ & $\begin{array}{l}\infty \\
\infty \\
\sigma\end{array}$ & 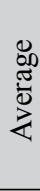 \\
\hline \multicolumn{2}{|c|}{ 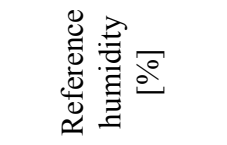 } & $\underset{\text { i }}{\text { i }}$ & $\begin{array}{l}8 \\
\text { i }\end{array}$ & $\begin{array}{l}8 \\
i\end{array}$ & $\begin{array}{l}8 \\
\text { i }\end{array}$ & $\begin{array}{l}8 \\
\text { i }\end{array}$ & & $\begin{array}{l}8 \\
\text { i }\end{array}$ & $\underset{8}{\stackrel{8}{1}}$ & $\begin{array}{l}8 \\
\text { i }\end{array}$ & $\begin{array}{l}8 \\
\text { I }\end{array}$ & $\begin{array}{l}8 \\
\text { i }\end{array}$ & $\begin{array}{l}8 \\
\text { i }\end{array}$ & $\begin{array}{l}8 \\
\text { i }\end{array}$ & \\
\hline \multicolumn{2}{|c|}{ 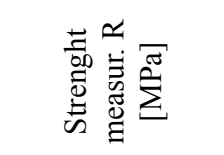 } & $\stackrel{n}{\stackrel{n}{6}}$ & @̊ & กิ่ & $\begin{array}{l}\text { D } \\
\text { in }\end{array}$ & $\stackrel{+}{\stackrel{t}{6}}$ & & $\begin{array}{l}\bar{\infty} \\
\infty \\
\dot{0}\end{array}$ & $\underset{n}{\tilde{\sigma}}$ & $\begin{array}{l}n \\
\vdots \\
a\end{array}$ & $\stackrel{n}{\stackrel{n}{\infty}}$ & $\begin{array}{l}n \\
\dddot{n} \\
\stackrel{2}{a}\end{array}$ & $\begin{array}{l}0 \\
n \\
\tilde{6}\end{array}$ & $\stackrel{\vec{J}}{\Xi}$ & \\
\hline \multicolumn{2}{|c|}{ 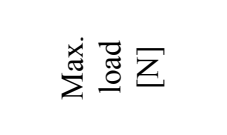 } & $\begin{array}{l}8 \\
8 \\
0 \\
0 \\
0 \\
0\end{array}$ & $\begin{array}{l}8 \\
8 \\
0 \\
0 \\
0\end{array}$ & 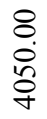 & 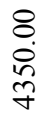 & $\begin{array}{l}8 \\
\stackrel{0}{0} \\
\dddot{y}\end{array}$ & & $\begin{array}{l}8 \\
8 \\
8 \\
0\end{array}$ & $\begin{array}{l}8 \\
\dot{8} \\
\infty \\
-\end{array}$ & $\begin{array}{l}8 \\
\stackrel{8}{8} \\
\stackrel{+}{+}\end{array}$ & $\begin{array}{l}8 \\
\circ \\
\stackrel{2}{2}\end{array}$ & $\begin{array}{l}8 \\
\stackrel{8}{0} \\
\stackrel{n}{N}\end{array}$ & $\begin{array}{l}8 \\
\dot{8} \\
\dot{8}\end{array}$ & 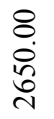 & \\
\hline \multicolumn{2}{|c|}{ 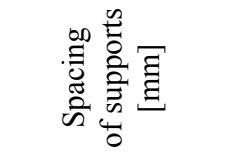 } & $\begin{array}{l}8 \\
\ddot{\circ} \\
\stackrel{2}{1}\end{array}$ & $\begin{array}{l}8 \\
8 \\
\stackrel{n}{2}\end{array}$ & $\begin{array}{l}8 \\
\circ \\
\stackrel{1}{2}\end{array}$ & $\begin{array}{l}8 \\
\dot{0} \\
i\end{array}$ & $\begin{array}{l}8 \\
\stackrel{0}{\circ} \\
i\end{array}$ & & $\begin{array}{l}8 \\
\dot{8} \\
\stackrel{i}{1}\end{array}$ & $\begin{array}{l}8 \\
\dot{8} \\
\stackrel{\leftrightarrow}{1}\end{array}$ & $\begin{array}{l}8 \\
\dot{8} \\
\stackrel{8}{1}\end{array}$ & $\begin{array}{l}8 \\
\dot{8} \\
\text { ¿ }\end{array}$ & $\begin{array}{l}8 \\
\dot{8} \\
\stackrel{8}{1}\end{array}$ & 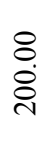 & $\begin{array}{l}8 \\
\stackrel{8}{0} \\
\stackrel{0}{0}\end{array}$ & \\
\hline \multirow{4}{*}{$\bar{z}$} & 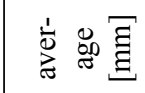 & $\begin{array}{l}\stackrel{0}{n} \\
\infty \\
\infty\end{array}$ & $\begin{array}{l}\stackrel{\sim}{1} \\
\infty \\
\sim\end{array}$ & $\begin{array}{l}\stackrel{n}{+} \\
\stackrel{\sim}{N}\end{array}$ & $\stackrel{\stackrel{?}{\sim}}{\stackrel{\sim}{\sim}}$ & $\stackrel{\stackrel{8}{\sim}}{\stackrel{\sim}{\sim}}$ & & $\stackrel{\bar{m}}{\stackrel{2}{2}}$ & $\stackrel{\circ}{2}$ & $\frac{1}{9}$ & $\stackrel{+}{\circ}$ & $\underset{\Xi}{\Xi}$ & $\stackrel{n}{\stackrel{2}{I}}$ & $\begin{array}{l}\infty \\
\tilde{m} \\
\infty \\
\infty\end{array}$ & \\
\hline & $\dot{\ddot{J}} n$ & $\underset{\substack{i \\
i}}{\stackrel{i}{i}}$ & $\underset{\infty}{\infty}$ & $\begin{array}{l}\hat{\sigma} \\
\stackrel{\sim}{N}\end{array}$ & 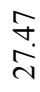 & $\begin{array}{l}n \\
\stackrel{2}{0} \\
\stackrel{i}{1}\end{array}$ & & $\begin{array}{l}\infty \\
\infty \\
\infty \\
\infty\end{array}$ & $\begin{array}{l}\circ \\
\infty \\
\infty\end{array}$ & $\stackrel{1}{9}$ & $\begin{array}{l}\stackrel{9}{9} \\
\stackrel{9}{-}\end{array}$ & $\hat{n}$ & $\begin{array}{l}\tilde{\sigma} \\
\infty \\
\infty\end{array}$ & $\begin{array}{l}\infty \\
\stackrel{\infty}{\Xi}\end{array}$ & \\
\hline & $\stackrel{\dot{\Xi}}{\Xi} \sim$ & $\begin{array}{l}\hat{\sigma} \\
\infty \\
\dot{\sim}\end{array}$ & $\begin{array}{l}\infty \\
\stackrel{\infty}{\sim} \\
\stackrel{\infty}{N}\end{array}$ & $\begin{array}{l}\infty \\
\stackrel{\infty}{\sim}\end{array}$ & $\begin{array}{l}8 \\
\infty \\
\stackrel{\sim}{0}\end{array}$ & 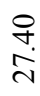 & & $\stackrel{\circ}{\stackrel{0}{9}}$ & $\begin{array}{l}\stackrel{2}{2} \\
\stackrel{2}{2}\end{array}$ & $\stackrel{ \pm}{a}$ & $\begin{array}{l}\tilde{\infty} \\
\infty \\
\infty\end{array}$ & $\stackrel{8}{\circ}$ & $\stackrel{5}{\Xi}$ & $\begin{array}{l}0 \\
\stackrel{\infty}{\sim}\end{array}$ & \\
\hline & $\stackrel{\dot{\Xi}}{\stackrel{\Xi}{\Xi}}-$ & $\begin{array}{l}0 \\
\stackrel{0}{\sim} \\
\sim\end{array}$ & $\begin{array}{l}\infty \\
\stackrel{\infty}{0} \\
\stackrel{\infty}{N}\end{array}$ & 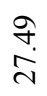 & 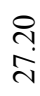 & 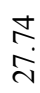 & & $\begin{array}{l}\infty \\
\stackrel{2}{2}\end{array}$ & $\hat{a}$ & $\frac{0}{9}$ & $\stackrel{\bar{N}}{\stackrel{2}{2}}$ & $=$ & $\hat{a}$ & $\stackrel{1}{2}$ & \\
\hline \multirow{4}{*}{$\begin{array}{l}= \\
1 \\
\frac{1}{60} \\
.9\end{array}$} & 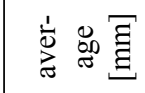 & $\underset{n}{\stackrel{n}{n}}$ & 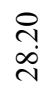 & $\frac{n}{\infty}$ & $\begin{array}{l}\text { oे } \\
\infty \\
\stackrel{\sim}{\sim}\end{array}$ & $\begin{array}{l}\text { o. } \\
\infty \\
\sim\end{array}$ & & $\begin{array}{l}\bar{\circ} \\
\stackrel{0}{-}\end{array}$ & 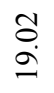 & $\begin{array}{l}\overrightarrow{0} \\
\stackrel{0}{0}\end{array}$ & $\underset{\infty}{\stackrel{0}{\infty}}$ & $\frac{\infty}{a}$ & ते & $\stackrel{R}{2}$ & \\
\hline & 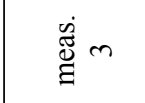 & 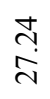 & $\begin{array}{l}\text { ¿ } \\
\stackrel{\infty}{\sim}\end{array}$ & $\stackrel{m}{\stackrel{\infty}{\sim}}$ & $\begin{array}{l}\stackrel{n}{\sim} \\
\stackrel{\infty}{\sim}\end{array}$ & $\begin{array}{l}\infty \\
\stackrel{\infty}{\sim}\end{array}$ & & $\begin{array}{l}8 \\
\infty \\
\infty\end{array}$ & $\begin{array}{l}\stackrel{\alpha}{\infty} \\
\infty\end{array}$ & $\begin{array}{l}8 \\
0 \\
9\end{array}$ & $\frac{n}{\infty}$ & $\vec{m}$ & $\begin{array}{l}\infty \\
\stackrel{\infty}{-}\end{array}$ & $\stackrel{2}{2}$ & \\
\hline & $\stackrel{\dot{\mathscr{J}}}{\stackrel{\Xi}{\Xi}} \sim$ & $\begin{array}{l}\infty \\
\stackrel{0}{\sim} \\
\stackrel{\sim}{N}\end{array}$ & $\begin{array}{l}\text { ते } \\
\stackrel{\sim}{N}\end{array}$ & $\frac{0}{\infty}$ & $\begin{array}{l}\text { ते } \\
\stackrel{\sim}{N}\end{array}$ & $\begin{array}{l}0 \\
+ \\
\infty \\
\text { N }\end{array}$ & & $\begin{array}{l}2 \\
\stackrel{2}{a}\end{array}$ & $\begin{array}{l}\Phi \\
\infty \\
\infty\end{array}$ & $\begin{array}{l}\tilde{6} \\
\stackrel{2}{a}\end{array}$ & $\begin{array}{l}\infty \\
\stackrel{\infty}{\infty}\end{array}$ & $\frac{ \pm}{a}$ & $\stackrel{?}{\stackrel{2}{2}}$ & $\begin{array}{l}\dot{\infty} \\
\stackrel{\infty}{\sigma}\end{array}$ & \\
\hline & $\stackrel{\dot{\Xi}}{\stackrel{\Xi}{\Xi}}-$ & $\frac{\widetilde{\sigma}}{\stackrel{\sim}{\tau}}$ & $\begin{array}{l}0 \\
\text { n} \\
\infty \\
\sim\end{array}$ & $\frac{n}{\infty}$ & $\stackrel{\vec{n}}{\stackrel{N}{N}}$ & 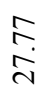 & & $\stackrel{\circ}{\circ}$ & సָ & $\begin{array}{l}\infty \\
\vdots \\
a\end{array}$ & 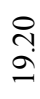 & $\stackrel{\infty}{\circ}$ & ๙ి & $\begin{array}{l}\infty \\
\stackrel{\leftrightarrow}{+}\end{array}$ & \\
\hline \multicolumn{2}{|r|}{$\dot{2}$} & - & $\nabla$ & in & 0 & $r$ & & - & N & $n$ & $n$ & $r$ & $\infty$ & 으 & \\
\hline & & \multicolumn{5}{|c|}{ Q səฺ̣əs } & & \multicolumn{7}{|c|}{ W səțəS } & \\
\hline \multicolumn{15}{|c|}{ КчңґәНН } & \\
\hline
\end{tabular}




\begin{tabular}{|c|c|c|c|c|c|c|c|c|c|c|c|c|c|c|c|}
\hline \multicolumn{2}{|c|}{ 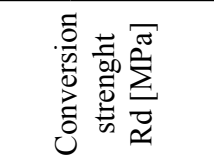 } & $\begin{array}{l}\text { fे } \\
\text { \&̊ }\end{array}$ & f̊ & $\begin{array}{l}\vec{\sigma} \\
\stackrel{2}{2}\end{array}$ & $\begin{array}{l}\text { ळे } \\
\text { ळे }\end{array}$ & $\frac{ \pm}{\dot{8}}$ & $\begin{array}{l}n \\
\hat{n} \\
i n\end{array}$ & $\begin{array}{l}\infty \\
\dot{0} \\
\stackrel{0}{0}\end{array}$ & $\begin{array}{l}\vec{b} \\
\infty \\
\infty\end{array}$ & 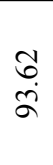 & $\underset{⿱ 宀}{\stackrel{8}{8}}$ & $\begin{array}{l}\bar{\infty} \\
\dot{\infty} \\
\infty\end{array}$ & $\begin{array}{l}0 \\
0 \\
0 \\
0\end{array}$ & $\begin{array}{l}\stackrel{J}{n} \\
\stackrel{\delta}{n}\end{array}$ & $=$ \\
\hline \multicolumn{2}{|c|}{ 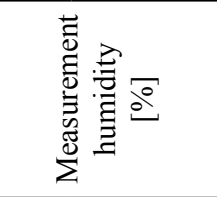 } & $\begin{array}{l}\infty \\
=\end{array}$ & $\stackrel{\infty}{\stackrel{\infty}{\beth}}$ & $\begin{array}{l}\stackrel{0}{i} \\
\stackrel{i}{c}\end{array}$ & $\stackrel{\tilde{\vartheta}}{\hat{0}}$ & $\stackrel{\overbrace{}}{=}$ & $\stackrel{n}{\varrho}$ & 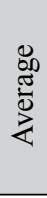 & $\begin{array}{l}\stackrel{0}{\circ} \\
\infty\end{array}$ & n̊? & $\stackrel{5}{\circ}$ & $\stackrel{\infty}{\stackrel{\infty}{r}}$ & $\underset{\infty}{\stackrel{+}{\infty}}$ & $\begin{array}{l}œ \\
\infty \\
\sigma\end{array}$ & 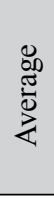 \\
\hline \multicolumn{2}{|c|}{ 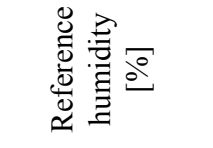 } & $\begin{array}{l}\stackrel{8}{ } \\
\text { i }\end{array}$ & $\begin{array}{l}\stackrel{8}{0} \\
\text { i }\end{array}$ & $\begin{array}{l}8 \\
i \\
\end{array}$ & $\begin{array}{l}8 \\
\text { i }\end{array}$ & $\begin{array}{l}8 \\
1 \\
1\end{array}$ & $\begin{array}{l}8 \\
\text { i }\end{array}$ & & $\begin{array}{l}8 \\
\text { i }\end{array}$ & $\begin{array}{l}8 \\
\text { i }\end{array}$ & $\begin{array}{l}8 \\
\text { i }\end{array}$ & $\begin{array}{l}8 \\
i\end{array}$ & $\begin{array}{l}8 \\
\text { i }\end{array}$ & $\begin{array}{l}8 \\
\text { i }\end{array}$ & \\
\hline \multicolumn{2}{|c|}{ 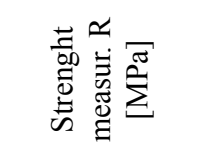 } & $\begin{array}{l}\hat{\infty} \\
\dot{a}\end{array}$ & $\stackrel{m}{n}$ & 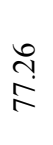 & $\begin{array}{l}\vec{\infty} \\
\dot{+}\end{array}$ & $\stackrel{n}{\pi}$ & $\stackrel{n}{i}$ & & $\stackrel{m}{\stackrel{2}{a}}$ & 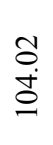 & $\begin{array}{l}8 \\
i \\
i\end{array}$ & $\stackrel{n}{\stackrel{n}{0}}$ & 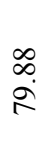 & $\frac{n}{6}$ & \\
\hline \multicolumn{2}{|c|}{$\stackrel{\dot{x}}{\stackrel{\Xi}{\Xi} \Xi}$} & $\begin{array}{l}8 \\
\dot{8} \\
\dot{0}\end{array}$ & $\begin{array}{l}8 \\
\stackrel{9}{8} \\
\dot{q}\end{array}$ & $\begin{array}{l}8 \\
\stackrel{0}{\circ} \\
\stackrel{\sigma}{\sigma}\end{array}$ & 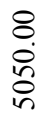 & 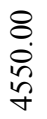 & $\begin{array}{l}8 \\
\text { \&ें } \\
\text { ले }\end{array}$ & & $\begin{array}{l}8 \\
\stackrel{0}{0} \\
\stackrel{1}{2}\end{array}$ & $\begin{array}{l}8 \\
8 \\
\stackrel{0}{0} \\
\text { त }\end{array}$ & $\begin{array}{l}8 \\
\stackrel{8}{8}\end{array}$ & $\begin{array}{l}8 \\
\stackrel{8}{0} \\
\stackrel{8}{+}\end{array}$ & $\begin{array}{l}8 \\
\stackrel{8}{8}\end{array}$ & $\begin{array}{l}8 \\
8 \\
8 \\
-1\end{array}$ & \\
\hline \multicolumn{2}{|c|}{ 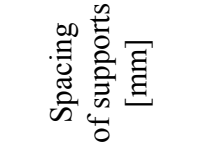 } & $\begin{array}{l}8 \\
\ddot{0} \\
\end{array}$ & $\begin{array}{l}8 \\
\ddot{1} \\
i\end{array}$ & $\begin{array}{l}8 \\
\ddot{1} \\
i\end{array}$ & $\begin{array}{l}8 \\
\ddot{+} \\
i \\
i\end{array}$ & $\begin{array}{l}8 \\
\stackrel{8}{1} \\
\end{array}$ & $\begin{array}{l}8 \\
\stackrel{0}{0} \\
\stackrel{2}{1}\end{array}$ & & $\begin{array}{l}8 \\
\stackrel{8}{8}\end{array}$ & $\begin{array}{l}8 \\
\stackrel{8}{8}\end{array}$ & $\begin{array}{l}8 \\
\stackrel{8}{0} \\
\stackrel{0}{1}\end{array}$ & $\begin{array}{l}8 \\
\stackrel{8}{0} \\
\stackrel{1}{0}\end{array}$ & $\begin{array}{l}8 \\
\stackrel{8}{0} \\
\stackrel{1}{1}\end{array}$ & $\begin{array}{l}8 \\
\stackrel{\leftrightarrow}{0}\end{array}$ & \\
\hline \multirow{4}{*}{$\begin{array}{l}0 \\
1 \\
\frac{1}{0} \\
0 \\
3\end{array}$} & 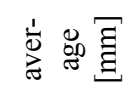 & 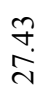 & $\stackrel{n}{\infty}$ & $\begin{array}{l}i \\
i \\
\infty \\
i\end{array}$ & $\begin{array}{l}\stackrel{8}{0} \\
\stackrel{\sim}{N}\end{array}$ & बें & i̊ & & શે̀ & $\begin{array}{l}\vec{\infty} \\
\stackrel{a}{a}\end{array}$ & $\stackrel{m}{m}$ & $\stackrel{7}{9}$ & $\ddot{n}$ & $\begin{array}{l}8 \\
\stackrel{0}{2}\end{array}$ & \\
\hline & $\dot{\mathscr{J}} n$ & مूे & $\begin{array}{l}\infty \\
\stackrel{\infty}{\infty} \\
\stackrel{\infty}{\sim}\end{array}$ & $\begin{array}{l}+ \\
\infty \\
\infty \\
\stackrel{\sim}{d}\end{array}$ & $\underset{\Delta}{\Delta}$ & $=$ & ì. & & $\frac{n}{2}$ & $\stackrel{t}{0}$ & $\stackrel{\infty}{\sim}$ & $\underset{\substack{\infty \\
\infty}}{\infty}$ & $\stackrel{\circ}{2}$ & $\stackrel{\hat{\imath}}{\stackrel{2}{a}}$ & \\
\hline & $\stackrel{\dot{\mathscr{J}}}{\stackrel{\Xi}{\Xi}} \sim$ & $\begin{array}{l}\text { bे } \\
\stackrel{\sim}{*}\end{array}$ & $\stackrel{n}{\stackrel{n}{\sim}}$ & $\hat{n}$ & $\begin{array}{l}\Delta \\
\stackrel{\Delta}{\Delta}\end{array}$ & 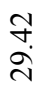 & in & & $\stackrel{n}{2}$ & $\stackrel{ }{a}$ & $\stackrel{\text { ֻे }}{2}$ & $\stackrel{\text { ते }}{\stackrel{2}{2}}$ & $\stackrel{n}{2}$ & 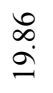 & \\
\hline & $\stackrel{\dot{m}}{\stackrel{\Xi}{\Xi}}-$ & $\begin{array}{l}\ddot{n} \\
\stackrel{\sim}{\sim}\end{array}$ & $\begin{array}{c}\tilde{\sim} \\
\infty \\
\sim\end{array}$ & $\begin{array}{c}\underset{0}{\infty} \\
\infty \\
\sim \\
\sim\end{array}$ & $\stackrel{\Re}{\stackrel{n}{\sim}}$ & $\begin{array}{l}\because 6 \\
\ddot{i}\end{array}$ & $\vec{m}$ & & $\stackrel{\circ}{\stackrel{0}{2}}$ & 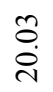 & $\stackrel{+}{\stackrel{\ddagger}{a}}$ & $\stackrel{?}{\stackrel{9}{2}}$ & $\stackrel{\dddot{\rho}}{9}$ & $\frac{1}{9}$ & \\
\hline \multirow{4}{*}{$\begin{array}{l}= \\
1 \\
\frac{50}{000} \\
.1\end{array}$} & 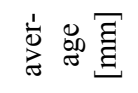 & $\stackrel{?}{\stackrel{?}{\sim}}$ & $\begin{array}{l}\stackrel{\infty}{\Delta} \\
\stackrel{\sim}{\sim}\end{array}$ & $\begin{array}{l}0 \\
\ddot{2}\end{array}$ & $\begin{array}{l}\text { \&े } \\
\text { ¿ें }\end{array}$ & $\begin{array}{l}\stackrel{i}{+} \\
\infty \\
i\end{array}$ & $\stackrel{\circ}{\stackrel{2}{*}}$ & & $\underset{\substack{n \\
\infty}}{\stackrel{n}{\infty}}$ & \begin{tabular}{l}
\multirow{0}{0}{} \\
$\stackrel{0}{-}$
\end{tabular} & $\begin{array}{l}\infty \\
0 \\
\infty \\
\infty\end{array}$ & $\stackrel{2}{\dddot{2}}$ & $\stackrel{-}{\infty}$ & $\stackrel{\infty}{\stackrel{\infty}{\Xi}}$ & \\
\hline & $\dot{\mathscr{J}} \mathrm{g}$ & $\stackrel{n}{\stackrel{n}{N}}$ & $\begin{array}{l}\infty \\
\infty \\
\stackrel{\sim}{\sim}\end{array}$ & ‡ั & 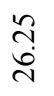 & 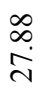 & ñ? & & $\begin{array}{l}\stackrel{0}{0} \\
\stackrel{0}{2}\end{array}$ & $\stackrel{+}{\sharp}$ & $\begin{array}{l}0 \\
n \\
\infty \\
-1\end{array}$ & $\hat{\sigma}$ & $\stackrel{?}{\stackrel{2}{I}}$ & 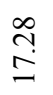 & \\
\hline & $\begin{array}{l}\dot{\mathscr{g}} \\
\dot{\Xi}\end{array}$ & $\stackrel{?}{\stackrel{?}{\sim}}$ & $\begin{array}{l}\stackrel{0}{\infty} \\
\infty \\
\stackrel{\infty}{\sim}\end{array}$ & $\begin{array}{l}\tilde{\alpha} \\
\infty \\
\infty\end{array}$ & $\begin{array}{l}\hat{\alpha} \\
\text { ¿े }\end{array}$ & 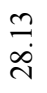 & $\begin{array}{l}\dot{0} \\
\stackrel{i}{0}\end{array}$ & & 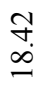 & $\stackrel{\infty}{\stackrel{+}{a}} \stackrel{0}{\stackrel{9}{a}}$ & $\begin{array}{l}\hat{n} \\
\infty\end{array}$ & $\begin{array}{l}\hat{\alpha} \\
\infty \\
-\infty\end{array}$ & $\begin{array}{l}\stackrel{\infty}{\perp} \\
\stackrel{-}{=}\end{array}$ & $\begin{array}{l}\Omega \\
\end{array}$ & \\
\hline & $\stackrel{\dot{\Xi}}{\stackrel{\Xi}{\Xi}}-$ & $\underset{\stackrel{i}{\sim}}{\stackrel{R}{\sim}}$ & $\underset{\stackrel{i}{i}}{\stackrel{2}{\sim}}$ & $\underset{\substack{\infty \\
\sim}}{\vec{N}}$ & $\begin{array}{l}\stackrel{\sim}{\sim} \\
\stackrel{\sim}{\sim}\end{array}$ & त्̀ & iे & & $\begin{array}{l}\stackrel{0}{0} \\
\stackrel{\infty}{-}\end{array}$ & $\begin{array}{l}\stackrel{8}{\circ} \\
\stackrel{\text { ¿ }}{ }\end{array}$ & $\begin{array}{l}\stackrel{\sigma}{\circ} \\
\stackrel{a}{a}\end{array}$ & $=$ & $\begin{array}{l}\bar{\sigma} \\
\infty \\
-\end{array}$ & 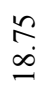 & \\
\hline \multirow{2}{*}{\multicolumn{2}{|c|}{$\dot{z}$}} & - & $m$ & 0 & $r$ & $a$ & 으 & & - & $m$ & $\nabla$ & 0 & $r$ & $\infty$ & \\
\hline & & \multicolumn{6}{|c|}{ Q sə̣ıəs } & & \multicolumn{6}{|c|}{ W səฺฺəs } & \\
\hline
\end{tabular}


Limbs of trees, called primary samples, from which next laboratory samples were prepared, were selected from fragments in the Krotoszyn Plateau area. Research material was collected from 60 -year-old pedunculate oaks (Quercus robur L.) forest stand. Trees were assessed according to the state of their crowns using defoliation and vitality evaluation. Defoliation expresses loss in the assimilative apparatus of assessed trees in relation to the image of a model tree that was not subjected to the impact of damage-causing factors. Defoliation evaluation of oaks was conducted in the middle

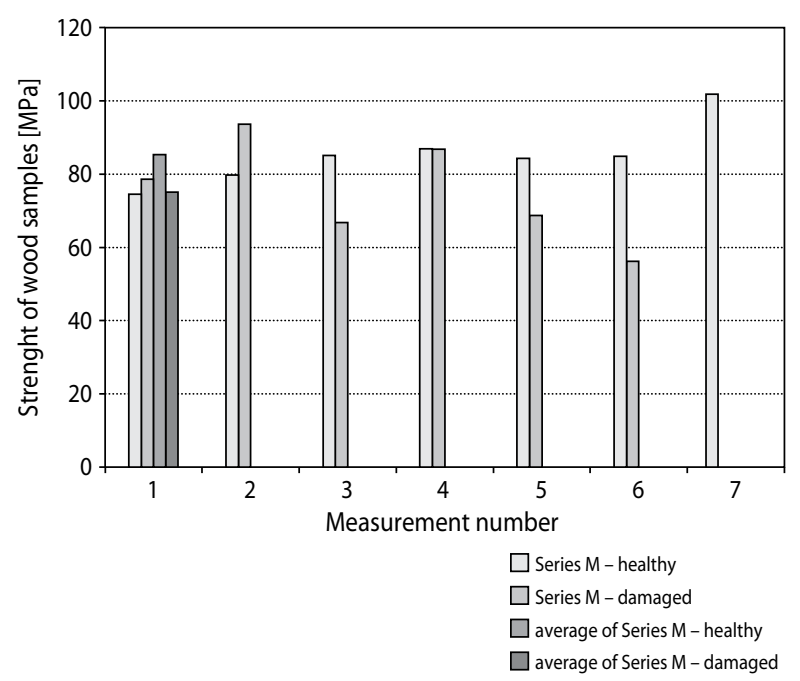

Figure 2. Results of samples strengh measurements in Series $\mathrm{M}-$ all (sample size $20 \times 20 \mathrm{~mm}$ )

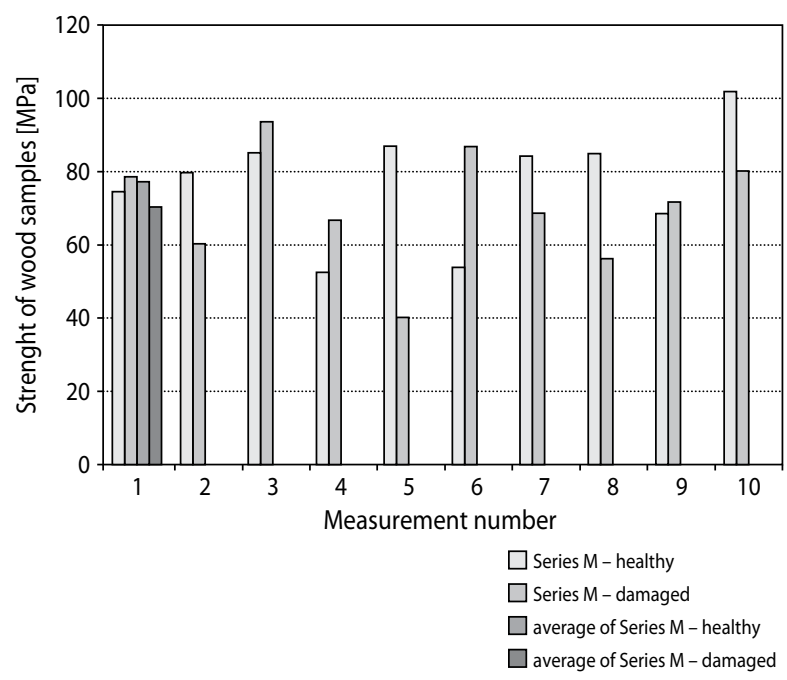

Figure 3. Results of samples strengh measurements in Series M - selected (sample size $20 \times 20 \mathrm{~mm}$ ) of June 2015. Another assessed parameter was vitality, which was assessed with a method developed by Andreas Roloff (1989). This parameter is representing the potential of tree growth and the ability to regenerate a damaged crown. The basis for vitality assessment is the system of shoots developed at the top of the crown. This evaluation includes not only the current state but also the changes in the deformation of shoots that occurred during the past few years. An explanation of the vitality classification is given in Table 3 . Weakened trees with defoliation exceeding $30 \%$ and a rate of vi-

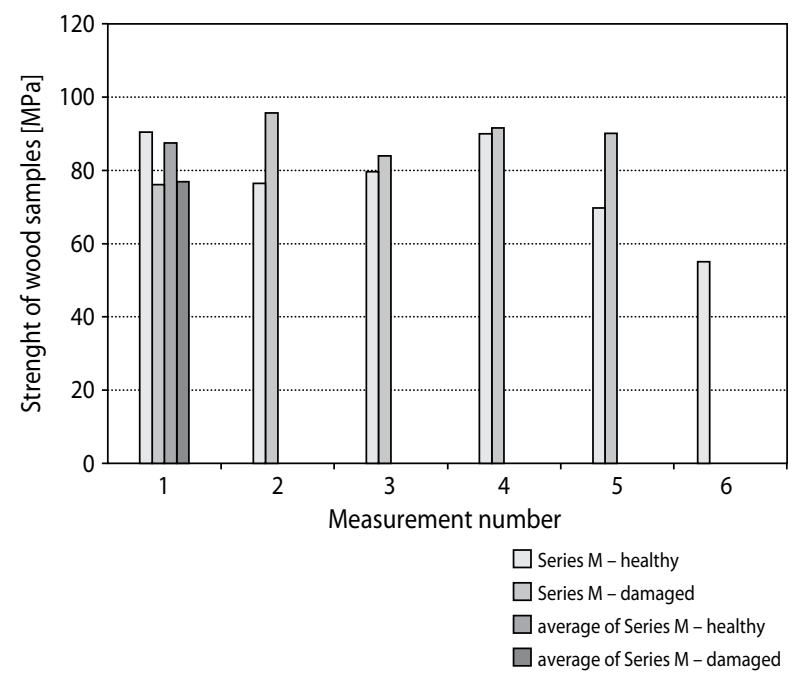

Figure 4. Results of samples strengh measurements in Series D - all (sample size $30 \times 30 \mathrm{~mm}$ )

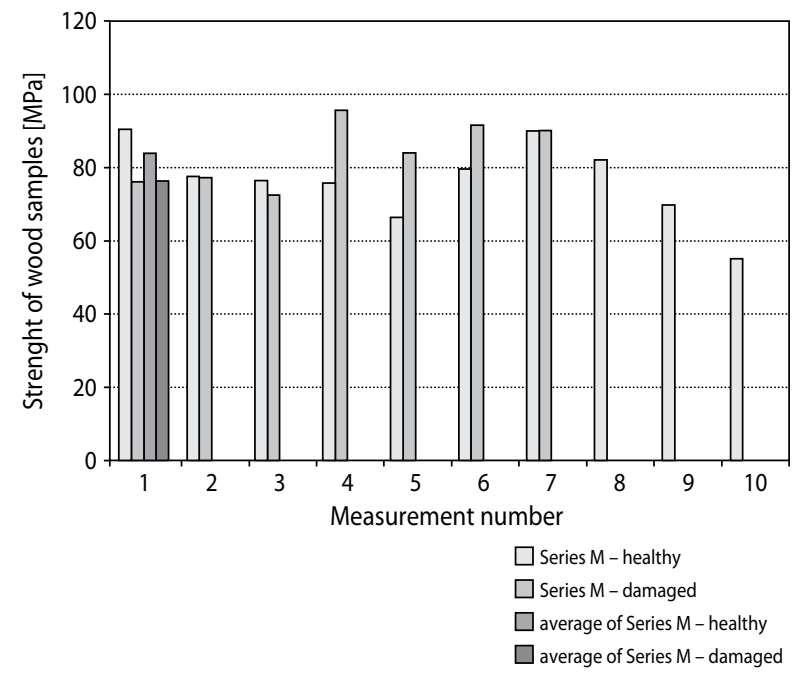

Figure 5. Results of samples strengh measurements in Series D - selected (sample size $30 \times 30 \mathrm{~mm}$ ) 
tality larger than 1 were considered diseased and damaged, and wood samples for testing were collected from those trees.

Table 3. Vitality degrees of oaks (according to Roloff's method)

\begin{tabular}{|l|l|c|}
\hline Vitality & \multicolumn{1}{|c|}{ Degree of damage } & $\begin{array}{c}\text { Number } \\
\text { of samples }\end{array}$ \\
\hline Degree 0 & $\begin{array}{l}\text { Exploration phase, undamaged, } \\
\text { vital trees }\end{array}$ & 7 \\
\hline Degree 1 & Degeneration phase, weakened trees & 10 \\
\hline Degree 2 & Stagnation phase, damaged trees & 10 \\
\hline Degree 3 & $\begin{array}{l}\text { Resignation phase, strongly } \\
\text { damaged, dying trees }\end{array}$ & 10 \\
\hline
\end{tabular}

After conducting the strength test, detailed measurements of humidity were performed by drying and weighing laboratory samples. Before measurement, samples were weighed and then dried at a temperature of $100^{\circ} \mathrm{C}$ for $10 \mathrm{~h}$, and then they were weighed again. In the next step, samples were additionally dried out, and the process was cyclically repeated in intervals of two hours until the constant weight and the difference not more than $0.002 \mathrm{~g}$. Results of measurements were presented in Figures 2-5.

\section{Results AND CONCLUSIONS}

Results of comparative analysis for all laboratory samples were presented in Figures 2-5. However, Figures 3 and 5 present the results of measurements of laboratory samples selected without visible norm defects according to the Polish Norm PN-EN 408+ A1 2012. Experimental and converse parameters are shown in Table 1 and for selected samples in Table 2. It is possible that in the case of Series M, samples No. 2, 4, 5 and 8 from damaged trees were rejected. Despite the selection of samples in the case of diseased trees, there is an increased amount of damaged timber structures relative to samples from healthy trees. The appearance of knots was noted in the plane of load, as was the local appearance of blue stain. A similar analysis was carried out for the samples of Series D. Owing to increased dimension, that is, $30 \mathrm{~mm}$ $\times 30 \mathrm{~mm}$ in the case of timber structures from damaged trees, it was difficult to collect samples without norm defects. In the Series D, 4 out of 10 samples collected from diseased trees were rejected, and 2 out of 7 samples collected from healthy trees were also rejected. In the case of Series D, the appearance of blue stain, brown algae, knots in the plane of load and defects in the structure of wood (i.e. turn of fibres and eccentric cores) were recorded.

Figure 2 presents the strength results obtained for the samples of Series M with a support span of $200 \mathrm{~mm}$ for all the tested samples. However, in Figure 3, results for selected samples without norm defects were presented. In opposite, the results for Series D with a support span of $250 \mathrm{~mm}$ were presented in Figures 4 and 5. It is possible that rejection of samples of healthy timber structures with norm defects in Series M caused an increase in average resistance to bending up to $8 \mathrm{MPa}$, and it causes an increase of up to $5 \mathrm{MPa}$ for timber structures of damaged trees in Series M. By analysing Series D, we can note that the increase was up to 3.5 $\mathrm{MPa}$ in the case of timber structures from healthy trees and $0.8 \mathrm{MPa}$ for the damaged trees.

The average strength of tested samples in Series M from healthy trees was $10 \mathrm{MPa}$ greater than those of samples from damaged trees, which makes $12 \%$ of the strength decrease. Similar decrease was recorded in the case of Series D.

The conducted strength tests showed that samples from trees with reduced health conditions indicated significant reduction in strength, compared with samples of healthy trees, going from $12 \%$ in the case of Series M and 13\% in the case of Series D. Samples of Series M and Series D showed that despite the differences in humidity and dimensions of the samples, the average results are repeatable. Individual differences in the results of samples are caused by diversity in timber structures. It should be stressed that in the cases of both diseased and healthy timber structures, there was selected research materials with a lack of norm defects, which is not possible in the case of latent defects in timber structures during inspection, and this had a significant impact on the final results. Figure 6 presents the structure of timber sample No. 10 of Series D, which had a deteriorated condition. It is possible that despite the appearance of norm defects, decrease in health conditions of the trees resulted in a comprehensive structural change of wood and thus affected the strength of the sample, for which a lower result was obtained in 
relation to the average of healthy samples (i.e. the biggest strength difference between damaged and healthy samples is $34 \%$ ). It was recorded that material collected directly from trees with deteriorated conditions indicated a much smaller preliminary humidity in relation to healthy trees.

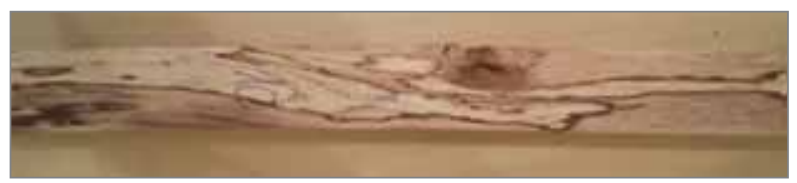

Figure 6. Sample No. 10 of Series D from trees with deteriorated conditions

\section{Acknowledgements}

This work was supported by Project HESOFF: 'Evaluation of the health state of forests and an effect of phosphite treatments with the use of photovoltaic SLE', Life 11 ENV/PL/459, co-financed by the European Community according to the LIFE + Program and by the National Fund for Environmental Protection and Water Management in Poland and Statutory Research Work no. 855 in the Military University of Technology.

\section{References}

Czech T., Hartmann G., Tomiczek C. 1998. Disease environment interactions in forest decline. In.: Disease/Environment Interactions in Forest Decline, Proceedings of a Workshop of the Working Party Disease/Environment Interactions in Forest Decline IUFRO 7.02.06, 16-21 March 1998, Vienna, Austria, 204.

Delatour C. 1983. The oak decline in Europe (in French). Revue Forestière Française, 35, 265-282.

Dreyer E., Aussenac G. 1996. Ecology and physiology of oaks in a changing environment. Annals of Forest Science, 53, 161-793.

Jung T., Blaschke H., Neumann P. 1996. Isolation, identification and pathogenicity of Phytophthora species from declining oak stands. European Journal of Forest Pathology, 26, 253-272.
Jung T., Cooke D.E.L., Blaschke H., Duncan J.M., Osswald W. 1999. Phytophthora quercina sp. nov., causing root rot of European oaks. Mycological Research, 103, 785-798.

Jung T., Hansen E.M., Winton L., Oßwald W., Delatour C. 2002. Three new species of Phytophthora from European oak forests. Mycological Research, 106, 397-411.

Oszako T. 2007. Causes of oak stand decline (in Polish with English summary). Sylwan, 6, 62-72.

Oszako T., Orlikowski L.B. 2005. First data on the occurrence of Phytophthora cinnamomi on pedunculate oak in Poland (in Polish with English summary). Sylwan, 149 (10), 47-53.

Oszako T., Orlikowski L.B., Trzewik A. 2007. Menace to polish forest nurseries by Phytophthora Species (in Polish with English summary). Progress in Plant Protection / Postępy w Ochronie Roślin, 47, 224-234.

Polish Norm PN-EN $408+$ A1. 2012. Drewno konstrukcyjne lite i klejone warstwowo. Oznaczanie niektórych właściwości fizycznych i mechanicznych. Polski Komitet Normalizacyjny, Warsaw.

Roloff A. 1989. Kronenentwicklung und Vitalitätsbeurteilung ausgewählter Baumarten der gemässigten Breiten. Schriften aus der Forstlichen Fakultät der Universität Göttingen und Niedersächsischen Forstlichen Versuchsanstalt, Frankfurt am Main.

Szymański E. 2003. Building Materials (in Polish). Wydawnictwa Szkolne i Pedagogiczne Spółka Akcyjna, Warsaw, Poland.

Vannini A. 1991. Hypoxylon mediterraneum: symptomatology and diffusion on Turkey oak in central Italy. In: Proceeding of an International Symposium „Oak Decline in Europe”, 15-18 May 1990, Kórnik, Poland, 159-164.

Vettraino A.M., Barzanti G.P., Bianco M.C., Ragazzi A., Capretti P., Paoletti E., Luisi N., Anselmi N., Vannini A. 2002. Occurrence of Phytophthora species in oak stands in Italy and their association with declining oak trees. Forest Pathology, 32, 19-28.

Wargo P.M. 1993. Multiple factors in oak decline in the United States. In: Recent advances in studies on oak decline, University of Bari Aldo Moro, Bari, Italy, 1-9. 